Article

\title{
Climatologia e Mudanças Climáticas: Avaliação da Produção Bibliográfica no Período de 2009 a 2019
}

\author{
Emerson Mariano da Silva ${ }^{1}$ (D), Francisco das Chagas Mesquita de Queiroz ${ }^{1}$, \\ José Maria Brabo Alves ${ }^{1}$ \\ ${ }^{1}$ Mestrado Profissional em Climatologia e Aplicações nos Países da CPLP e África, Universidade \\ Estadual do Ceará, Fortaleza, CE, Brasil.
}

Recebido em: 18 de Fevereiro de 2021 - Aceito em: 10 de Abril de 2021

\begin{abstract}
Resumo
Apresentam-se nesse trabalho os resultados de uma investigação realizada sobre a produção bibliográfica, no período de 2009 a 2019, nas áreas temáticas de climatologia e mudanças climáticas, disponível no Portal de Periódicos da CAPES, uma base de pesquisa de acesso gratuito para os membros da Comunidade Acadêmica Federada (CAFe) e Rede Nacional de Pesquisa (RNP) do Brasil. A produção bibliográfica investigada faz parte da área de Meteorologia que por sua vez está contida na área de Geociências da grande área do conhecimento de Ciências Exatas e da Terra, definidas pelo CNPq. Foram realizadas pesquisas nas bases indexadoras de periódicos (Science Direct-Elsevier, Scopus e SciELO) contidas no portal de periódicos usando-se os termos específicos "climatology", "climatology" and "Brazil", "climate change" e "climate change" and "Brazil", bem como a classificação dos periódicos que contém artigos com esses termos através do sistema brasileiro de classificação de periódicos (Qualis CAPES). Os resultados mostraram que 67\% dos periódicos dedicados às áreas de Meteorologia e Climatologia que agregam as publicações na temática de Mudanças Climáticas encontra-se no estrato de excelência (A) do Qualis CAPES.
\end{abstract}

Palavras-chaves: climatologia, mudanças climáticas, publicação bibliográfica.

\section{Climatology and Climate Change: Evaluation of Bibliographic Production in the period from 2009 to 2019}

\begin{abstract}
This work presents the results of an investigation carried out on bibliographic production, in the period from 2009 to 2019, in the thematic areas of climatology and climate change, available on the CAPES Journal Portal, a free access research base for members the Federated Academic Community (CAFe) and the National Research Network (RNP) of Brazil. The bibliographic production investigated is part of the Meteorology area, which in turn is contained in the Geosciences area of the large area of knowledge of Exact and Earth Sciences, defined by CNPq. Research was carried out on the indexing bases of journals (Science Direct-Elsevier, Scopus and SciELO) contained in the journals portal using the specific terms "climatology", "climatology" and "Brazil", "climate change" and "climate change" and "Brazil", as well as the classification of journals that contain articles with these terms through the Brazilian journal classification system (Qualis CAPES). The results showed that $67 \%$ of the journals dedicated to the areas of Meteorology and Climatology that aggregate publications on the theme of Climate Change are in the stratum of excellence (A) of Qualis CAPES.
\end{abstract}

Keywords: climatology, climate change, bibliographic production.

\section{Introdução}

A preocupação com clima e suas variabilidades ou mudanças está noticiada cotidianamente nos principais veículos de comunicação em todas as partes do mundo. Os desastres naturais associados a essas variabilidades e/ou as mudanças climáticas ganham visibilidade diariamente na imprensa falada, televisada, escrita e nas redes sociais. No entanto, a sociedade mundial ainda não dispõe de um processo de ampla divulgação dos resultados dos estudos científicos desenvolvidos nessas temáticas.

Autor de correspondência: Emerson Mariano da Silva. E-mail: emerson.mariano@uece.br. 
Visando melhorar o acesso da sociedade, e em particular das comunidades universitárias, as publicações científicas mundiais, o governo brasileiro lançou em novembro do ano 2000 o Portal de Periódicos da Capes, seguindo uma tendência mundial de lançamento das bibliotecas virtuais, com o processo de digitalização dos acervos de várias editoras nacionais e internacionais.

A proposta do portal de periódicos da CAPES foi usar o avanço tecnológico da época para possibilitar a comunidade científica o acesso livre e gratuito aos periódicos científicos nacionais e internacionais. Pois, o acesso aos periódicos científicos, em geral, era ou ainda é restrito a um número menor de cientistas que contam com financiamentos públicos e privados para suas pesquisas. Assim, uma nova modalidade de difusão da produção científica brasileira que passaria a fomentar democraticamente o desenvolvimento da ciência no país.

As publicações científicas nas temáticas em estudo fomentam as práticas de ensino e pesquisa acadêmica na área de Climatologia, uma subárea da Meteorologia, que por sua vez está contida na área de Geociências da grande área do conhecimento denominada de Ciencias Exatas e da Terra (Tabela de áreas do Conhecimento do Conselho Nacional de Desenvolvimento Científico e Tecnológico CNPq).

O objetivo desse estudo é apresentar, quantificar e qualificar a produção bibliográfica e os periódicos disponíveis nas bases indexadoras (Science Direct-Elsevier, Scopus e SciELO) contidas no Portal de Periódicos da CAPES (Coordenação de Aperfeiçoamento de Pessoal de Ensino Superior) que possibilitam a publicação de resultados dos estudos científicos desenvolvidos nessas temáticas.

Assim, o estudo também visa contribuir para a divulgação do acesso gratuito a esses conteúdos que pode fomentar novas buscas na produção de futuros trabalhos académicos e científicos.

\section{Breve Histórico das Publicações em Periódicos e a Descrição do Portal de Periódicos da CAPES}

Nos conta a história que na Grécia antiga as publicações das pesquisas seguiam a tradição comunicada em forma escrita, a fala e a escrita constituíam as formas mais importantes da comunicação científica na época. O processo de comunicação científica era bastante lento e perdurou por séculos, pois os textos eram transcritos manualmente e a comunicação era local.

Segundo Trevisol Neto (2017), citado em Ziman (1979), destaca-se a importância das sociedades reais e academias nacionais no desenvolvimento da ciência. Assim, é creditada à Royal Society de Londres a iniciativa de estimular a comunicação entre os cientistas, processo esse que teve início com as reuniões científicas e com a utilização de atas para informar e lembrar os participantes das questões abordadas nos encontros. Dessa forma, a uti- lização e troca de atas entre os membros das associações científicas originou os primeiros periódicos científicos.

A comunicação científica é processual e está suscetível a revisão dos pares, mecanismo em vigor há mais de 350 anos, que sem conhecer o autor de um artigo científico, especialistas avaliam de forma crítica o texto original, o método científico, a discussão dos resultados e das conclusões apresentadas, assim, emitem pareceres de aprovação ou não da possível publicação. Esse processo objetiva garantir a confiabilidade dos textos publicados que servirão de base para novas produções científicas em uma determinada área do conhecimento (Meadows, 1999, citado por Trevisol Neto, 2017).

A publicação de um artigo em periódico científico permite uma visibilidade maior do(a) pesquisador(a) ou de um grupo de pesquisa junto à comunidade científica dos seus pares, e pode servir de referência para a produção de outros artigos.

Segundo a Royal Society de Londres seriam quatro as funções do periódico científico: Comunicação formal dos resultados da pesquisa original para a comunidade científica e demais interessados, preservação e garantia da possibilidade de acesso aos conhecimentos publicados ao longo do tempo, estabelecimento da propriedade intelectual e a manutenção do padrão na qualidade na ciência (Campello et al., 1993 e Campello, 2000).

Campello (2000) também menciona que a divulgação científica no país apresenta uma série de problemas associados com a proliferação de periódicos que causa a dispersão da publicação de artigos sobre um mesmo assunto entre muitos títulos o que eleva em demasia o custo de atualização de coleções impressas. Em adição, cita que o periódico científico em meio eletrônico surgiu como alternativa inovadora e satisfatória para mitigar os problemas surgidos na logística de distribuição dos periódicos impressos, com rapidez na comunicação, flexibilidade de acesso, baixo custo relativo e disponibilidade imediata, capaz de diminuir os custos de atualização das coleções impressas.

De acordo com Russell (2001), citado por Mugnaini et al. (2019), o desenvolvimento tecnológico da informação viabilizou o acesso aos periódicos eletrónicos nos anos 1990. Esse acesso era possível quase exclusivamente de forma paga, um problema que representou grandes dificuldades para pesquisadores de países em desenvolvimento, que apesar de se beneficiarem do formato eletrônico, e disponibilidade online, se depararam com outras barreiras, que implicavam em altos custos e direitos autorais.

$\mathrm{Na}$ sequência temporal observou-se um movimento mundial de acesso gratuito aos periódico eletrônico, por meio do acesso remoto e de ferramentas de busca na internet, disponível nas universidades.

O Brasil se inseriu nesse contexto, com a criação do Portal de Periódicos da CAPES e da SciELO (Scientific 
Electronic Library Online) que foram criados com o objetivo de implementar bibliotecas virtuais capaz de fornecer acesso completo e gratuito aos conteúdos publicados eletronicamente em periódicos científicos, bancos de teses e dissertações, bem como a relatórios, manuais, dicionários, e a conteúdos audiovisuais.

\subsection{O Portal de Periódicos da CAPES}

O Portal de Periódico da CAPES é uma biblioteca virtual de informação científica de acesso gratuito que visa fomentar a comunidades científica com as principais publicações científicas do país e do mundo através de parcerias com importantes bases indexadoras de periódicos.

Ressalta-se que no portal eletrónico estão disponíveis 8.038 (oito mil e trinta e oito) títulos de periódicos com texto completo, 130 (cento e trinta) bases de dados de referências e resumos, 41 (quarenta e uma) bases de dados estatísticos, 64 (sessenta e quatro) bases de teses e dissertações, 48 (quarenta e oito) obras de referência dentre dicionários especializados, acervos especiais de bibliotecas, compêndios, bancos de dados e ferramentas de análise, 15 (quinze) bases de conteúdos audiovisuais, 14 (quatroze) bases de arquivos abertos e redes de e-prints, 12 (doze) bases de patentes, 2 (duas) bases de dados de normas técnicas e mais de 275.000 (duzentos e setenta e cinco mil) documentos dentre anais, relatórios, livros, anuários, guias, manuais dentre outros (Portal de Periódicos da Capes).

A Coordenação de Aperfeiçoamento de Pessoal de Nível Superior (CAPES) promove o acesso remoto ao Portal de Periódicos para os usuários vinculados às instituições participantes da rede da Comunidade Acadêmica Federada (CAFe). A Comunidade Academica Federada atua com a Rede Nacional de Pesquisa no desenvolvimento da ciência. O público constitui-se de estudantes da graduação e pós-graduação, professores universitários, comunidade acadêmica e profissionais da área de publicação.

O Qualis da CAPES, que constitui em um sistema brasileiro de avaliação de periódicos, busca contornar este problema, ao possibilitar que as áreas valorizem os periódicos não indexados, mas nem sempre consideram o volume de artigos publicados nos mesmos (Mugnaini et al., 2019).

\subsubsection{As bases indexadoras do Portal de Periódicos da CAPES}

Lancaster (2004) define uma base de dados bibliográficos pela sua utilidade ao responder as necessidades de informação, de acordo com quatro critérios principais: Cobertura, mostra o quanto completo é o conteúdo da base de dados em relação a um assunto; Recuperação, mostra uma estratégia de busca menos complexa; Previsibilidade, mostra a relevância de uma publicação a partir das informações contidas na base de dados; Atualidade, medida da velocidade com que novas publicações são incluídas na base. No portal de periódicos da CAPES encontram-se três grandes bases indexadoras de periódicos, descritas a seguir.

\subsubsection{Scopus}

É a maior base de resumos e citações da literatura revisada por pares no Mundo. Contém, entre outros, periódicos científicos, livros e anais de congressos. Oferece uma visão abrangente da produção mundial de pesquisa nas áreas de ciência, tecnologia da informação e comunicação, medicina, ciências sociais e artes e humanidades, a Scopus apresenta ferramentas inteligentes para rastrear, analisar e visualizar pesquisas, oferece acesso inigualável e contínuo a resultados de pesquisas científicas de todo o mundo. Além da informação, apresenta as ferramentas necessárias para analisá-las (Fonte: Scopus - Guia de Referência Rápida).

A Scopus oferece recursos gratuitos para usuários não inscritos e está disponível no Scopus Preview. Os pesquisadores podem usar os recursos para auxiliar em suas pesquisas, como pesquisar autores e aprender mais sobre a cobertura de conteúdo e as métricas de fonte da base (Fonte: Scopus - Guia de Referência Rápida).

Para acompanhar o que está acontecendo no mundo da pesquisa pode-se recorrer as ferramentas disponíveis e obter informações atualizadas em todos os campos de pesquisa: Ciências, matemática, engenharia, tecnologia, saúde e medicina, ciências sociais e artes e humanidades. Assim, o Scopus oferece uma ampla visão de informações científicas globais e interdisciplinares de que pesquisadores, professores e estudantes precisam para se manter informados (Fonte: Scopus - Guia de Referência Rápida).

\subsubsection{Science Direct}

A Elsevier é uma empresa global de informações analíticas que contribui com instituições e profissionais para o progresso da assistência à saúde e da ciência melhorando seu desempenho em benefício da humanidade. Atua como fonte de informação, auxilia os pesquisadores em novas descobertas e colaboração entre os pares, além de proporcionar o conhecimento necessário para que encontrem financiamento para sues projetos e pesquisas. Presta serviço para governos e universidades, além de avaliar e melhorar suas estratégias de pesquisa e ensino, com o objetivo de expandir as fronteiras do conhecimento para benefício da humanidade (Droescher e Silva, 2015).

Science Direct é a base de dados de texto completo e revisado por pares da Elsevier com aproximadamente 26.000 livros e mais de 2.200 periódicos. Além de buscas

sofisticadas e ferramentas para recuperação de resultados, inclui conteúdo integrado de várias fontes externas na forma de áudio, vídeo e conjuntos de dados (Fonte: Science Direct - Guia de Referência Rápida). 


\subsubsection{SciELO}

A SciELO é uma biblioteca eletrônica que abrange uma coleção selecionada de revistas científicas no idioma português, espanhol e outros, é parte integrante de um projeto desenvolvido pela Fundação de Amparo à Pesquisa do Estado de São Paulo (FAPESP), em parceria com o Centro Latino-Americano e do Caribe de Informação em Ciências da Saúde (BIREME), desde 2002, o projeto também é apoiado pelo Conselho Nacional de Desenvolvimento Científico e Tecnológico - CNPq (Fonte: Scielo).

O projeto prevê o desenvolvimento de uma metodologia comum para a preparação, armazenamento, disseminação e avaliação da literatura científica em formato eletrônico, fornecendo acesso total a uma coleção de títulos em série, a uma coleção de edições de títulos seriais individuais, bem como ao texto completo dos artigos, disponíveis através de índices e formulários eletrónicos de pesquisa (Fonte: Scielo).

\subsection{Estudos de Avaliação da Produção Bibliográfica Disponível no Portal de Periódicos da CAPES}

Ao fazer uma busca avançada no portal de periódicos da CAPES com os termos que remetem a avaliação da produção bibliográfica não se encontram artigos publicados na área de Meteorologia ou na subárea de Climatologia, ou ainda na temática das Mudanças Climáticas. Porém, encontram-se vários artigos publicados, em diversas áreas do conhecimento que quantificam e qualificam as produções bibliográficas em áreas específicas.

Freitas et al. (2017) avaliaram para a área de Ciências Sociais Aplicadas o número de artigos, citações e os indicadores de impacto dos periódicos disponíveis no SciELO, no período de 2009 a 2016. Assim, apontam que os artigos publicados nessa área do conhecimento têm maior visibilidade no Scopus que no Web of Sciense, e citam que isso se deve a política editorial e de indexação dessa base, que proporciona publicações em vários idiomas.

Puccini et al. (2015) mencionam que as bases mais importantes como fonte de pesquisa de artigos para a comunidade biomédica latino-americana, são a PubMed, o SciELO, além do Google Acadêmico. Assim, fizeram uma análise qualiquantitativa das publicações na área de Educação Médica e concluíram que para essa área os artigos publicados encontram maior visibilidade em periódicos que estão disponíveis no Google Acadêmico.

Erdmann et al. (2009), identificaram, quantificaram e qualificaram a produção bibliográfica para a área de Enfermagem em revistas nacionais com o objetivo de orientar a comunidade acadêmica para a seleção de periódicos e para o planejamento futuro dos programas de pósgraduação dessa área do conhecimento.

Trabalho semelhante aos descritos anteriormente foi realizado por Oliver (2008) na caracterização da produção bibliográfica na área de Terapia Ocupacional. Concluindose que para o período de 2001 a 2007, a maioria (41,7\%) dos 348 (trezentos e quarenta e oito) artigos publicados nessa área do conhecimento foram em periódicos nacionais, classificados no extrato B do Qualis CAPES, sendo $4,6 \%$ do total dos artigos publicados em periódicos classificados no extrato A do Qualis CAPES.

Ressalta-se que, apesar de não se encontrar artigos publicados nessa temática para a área de Meteorologia e em específico para a subárea de Climatologia, os artigos encontrados com as análises para as outras áreas do conhecimento nortearam as análises quantitativas e qualitativas apresentadas nesse estudo.

\section{Materiais e Métodos}

O percurso metodológico realizado nas pesquisas dos artigos relacionados a temática em estudo nas bases indexadoras de periódicos contidas no Portal de Periódicos da CAPES (Science Direct, Scopus e SciELO) é mostrado na Fig. 1. Foram realizadas as pesquisas nas três bases indexadoras usando-se os termos de busca "climatology", "climatology" and "Brazil", "climate change" e "climate change" and "Brazil".

Os resultados obtidos nas pesquisas realizadas, com período definido de 2009 a 2019, retornam as informações das publicações (título e resumo) da última década a partir das palavras-chaves que representam os estudos em climatologia e mudanças climáticas, assim, possibilita a posterior classificação dos documentos recuperados em: artigo científico e de revisão, capítulo de livros, resumos, resenha de livros, relatos de casos, correspondência, artigo de dados, entre outros.

Esses periódicos (nacionais e internacionais) são qualificados pelo sistema brasileiro de avaliação de periódicos da Coordenação de Aperfeiçoamento de Pessoal de Nível Superior (CAPES), denominado de Qualis CAPES. Assim, os resultados apresentados a seguir na seção 4.4, mostram a qualificação dos periódicos mais acessados para publicações, bem como a dos potenciais periódicos para futuras publicações dos artigos científicos por pesquisadores brasileiros que dedicam seus estudos e pesquisas as temáticas de climatologia e de mudanças climáticas.

O Qualis CAPES é divido por extratos que de vai de $\mathrm{A}$ (A1 e A2) a C, com o extrato B apresentando cinco subdivisões (B1, B2, B3, B4 e B5), e pode ser acessado de forma gratuita usando-se o endereço eletrônico da Plataforma Sucupira da CAPES, clicando-se no ícone Qualis. Menciona-se que existem dois eventos classificatórios de periódicos na página do Qualis e que para esse estudo adotou-se a classificação mais recente, denominada de evento 2013-2016, especificamente para a área de Geociências, em que está inserida a área de Meteorologia com a subárea de Climatologia que agrega os estudos na temática de Mudanças Climáticas. 


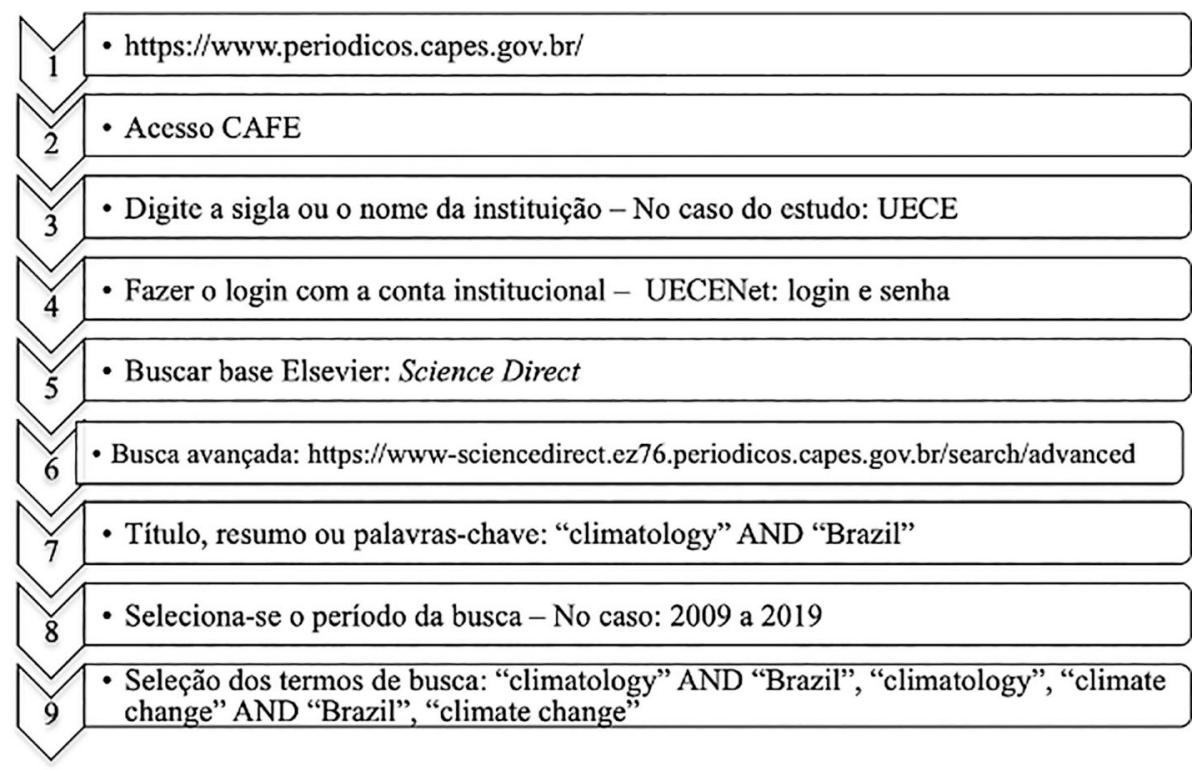

Figura 1 - Método de pesquisa Science Direct (Elsevier).

\section{Resultados e Discussões}

4.1. Resultados obtidos na base Science Direct

Os resultados mostram o crescimento anual do total de publicações que contém os termos "climatology" e "climatology" and "Brazil", no período de 2009 e 2019 (Fig. 2). Observam-se os totais de 2.134 e de 42 publicações no período, respectivamente.

A tipificação das publicações que contém os termos "climatology" and "Brazil" é mostrada na Fig. 3. Os resultados mostram que os capítulos de livro correspondem a $2,4 \%$ das publicações, os artigos de revisão totalizam 4,8\% das publicações e os artigos que apesentam resultados de pesquisa científica correspondem a $92,9 \%$ das publicações.

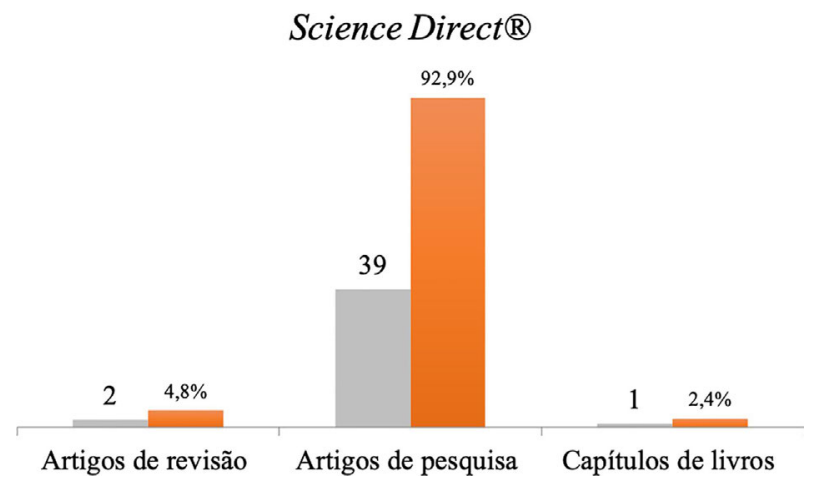

Figura 3 - Tipificação das publicações encontradas com o termo "climatology" and "Brazil" (2009-2019) - Science Direct. Fonte: Portal de Periódicos da Capes (2020).

\section{Science Direct ${ }^{\circledR}$}

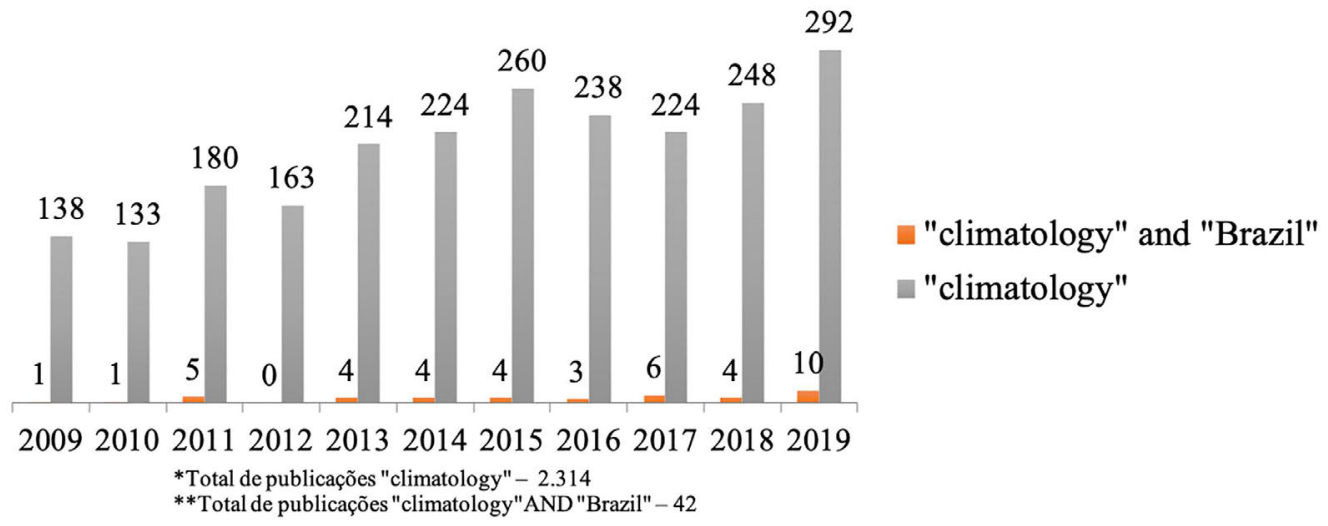

Figura 2 - Resultados obtidos usando-se os termos "climatology" and "Brazil" e "climatology" (2009-2019) - Science Direct. Fonte: Portal de Periódicos da Capes (2020). 
(a)

Science Direct ${ }^{\circledR}$
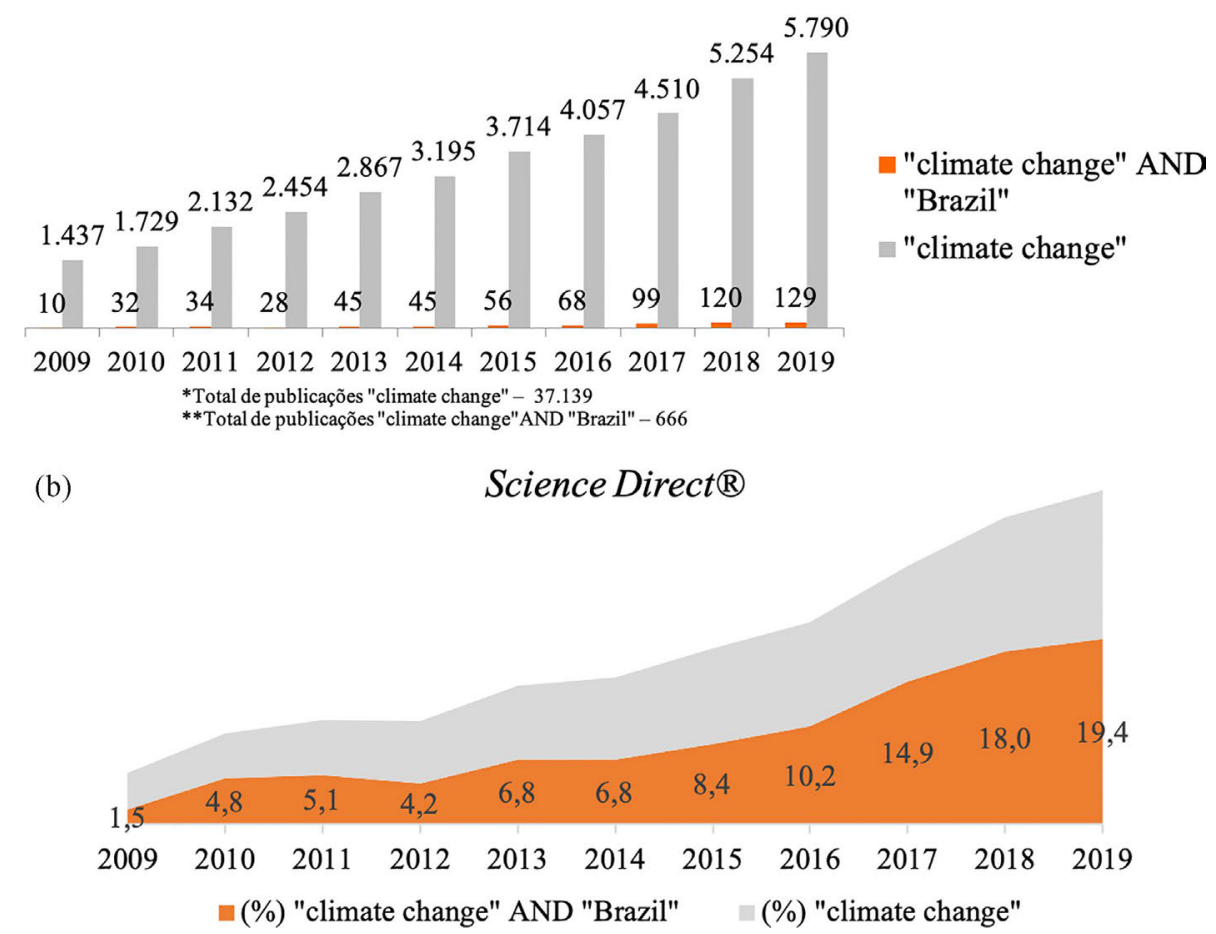

Figura 4 - Resultados obtidos usando-se os termos "climate change" and "Brazil" e "climate change" (2009-2019) - Science Direct. Fonte: Portal de Periódicos da Capes (2020).

A hipótese é que o grande número de publicações encontrada nessa modalidade (artigo científico) tenha relação com a necessidade de melhoria na classificação de pesquisadores e de programas de pós-graduação brasileiros, classificação que é baseada no índice de qualidade dos periódicos (Qualis CAPES). Alta pontuação contribui para a obtenção da aprovação de fomento para as futuras pesquisas e para o financiamento de futuras publicações.

A Fig. 4 mostra o número total de publicações pesquisadas com os termos "climate change" e "climate change" and "Brazil", no período de 2009 a 2019. Os resultados mostram o maior número de publicações na área temática de mudanças de climáticas em relação aos encontrados para a área de climatologia, tanto para o termo "climate change", que apresenta 37.139 (trinta e sete mil cento e trinta e nove) publicações, como para o termo "climate change" and "Brazil", que apresenta 666 (seiscentos e sessenta e seis) publicações, mostrados na Fig. 4a.

Em relação ao percentual de publicações ao longo do período em estudo (Fig. 4b), observa-se um crescimento ao longo do período de $1,5 \%$ das publicações em 2009 a 19,4\% das publicações em 2019.

A tipificação das publicações e os percentuais encontrados em cada modalidade de publicação são mostrados na Fig. 5. Os resultados mostram diversas formas (modalidades) de publicações que são usadas por pesqui- sadores que investigam as questões relacionadas as mudanças climáticas no país. Encontram-se, desde artigos (científicos e de revisão), que juntos totalizam $94,2 \%$ das publicações, o restante está distribuído entre livros, capítulos de livros, resumos (simples e expandido), editoriais, enciclopédia, entre outras.

Novamente, assim como para área de climatologia tem-se o maior número de publicações encontradas em forma de artigos em periódicos e que descrevem resultados de pesquisas científicas, fato que corrobora com a hipótese levantada na análise anterior (Qualis CAPES).

\subsection{Resultados obtidos na base Scopus}

Os resultados apresentados na Fig. 6a mostram 41.149 (quarenta e uma mil e cento e quarenta e nove) publicações encontradas nessa base usando-se o termo "climatology" e 682 (seiscentos e oitenta e duas) publicações quando se usa os termos "climatology" and "Brazil". Destaca-se que são resultados mais expressivos que os encontrados na base Science Direct da Elselvier: 2.134 (duas mil e cento e trinta e quatro) publicações ("climatology") e 42 (quarenta e duas) publicações ("climatology" and "Brazil").

Em relação a evolução do percentual (\%) de publicação na área temática de climatologia no país ("climatology" and "Brazil"), mostrada na Fig. 6 b, destaca-se o 


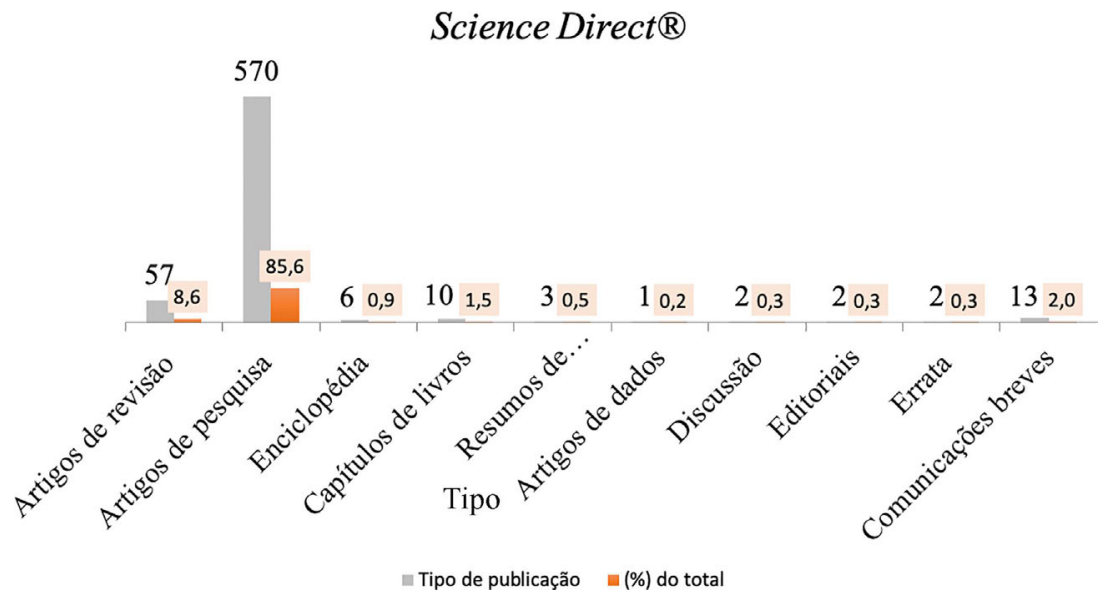

Figura 5 - Tipificação das publicações encontradas na pesquisa com o termo "climate change" and "Brazil" (2009-2019) - Science Direct. Fonte: Portal de Periódicos da Capes (2020).

(a)

\section{Scopus ${ }^{\circledR}$}

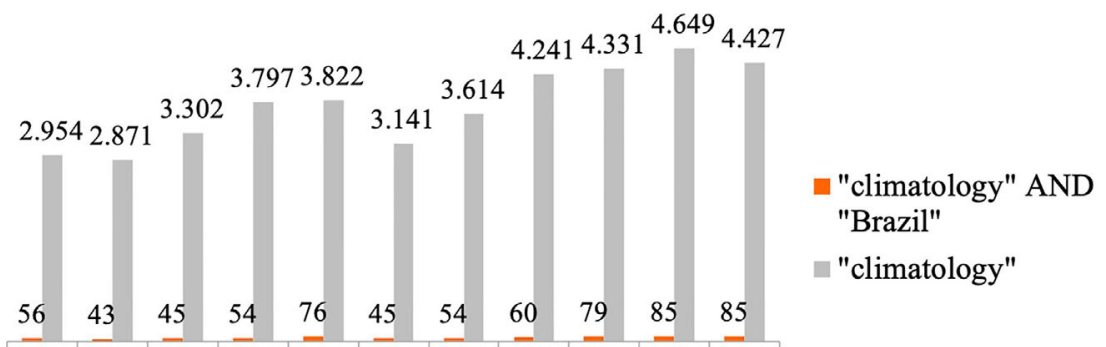

20092010201120122013201420152016201720182019

*Total de publicações "climatology"- 41.149

**Total de publicaçōes "climatology"AND "Brazil" -682

(b) Scopus $\mathbb{R}$

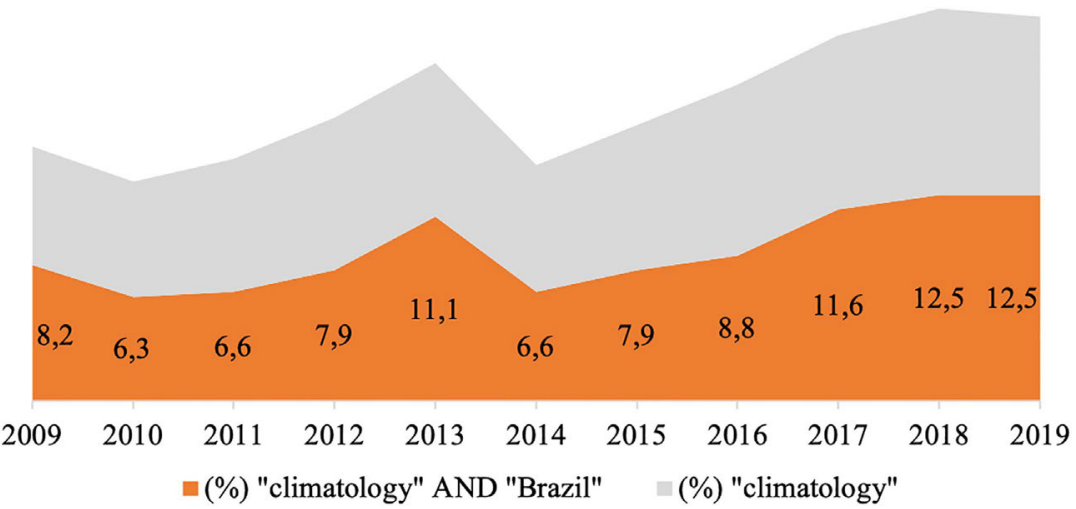

Figura 6 - Resultados obtidos usando-se os termos "climatology" and "Brazil" e "climatology" (2009-2019) - Scopus. Fonte: Portal de Periódicos da Capes (2020).

período entre 2016 e 2019 com percentuais que variam de 8,8 a $12,5 \%$ do total de publicações em cada um desses anos, totalizando 45,4\% das publicações na área encontradas na base Scopus.

Os quantitativos das publicações usando-se o termo "climate change" totalizam 209.216 (duzentos e nove mil e duzentos e dezesseis) no período e usando-se os termos "climate change" and "Brazil" encontram-se 3.177 (três mil cento e setenta e sete) publicações nessa base indexadora. Destaca-se que são valores aproximadamente seis vezes maiores do que os encontrados com esses termos na base Science Direct (Fig. 7a). 
Os percentuais (\%) anuais de publicações apresentados na Fig. $7 \mathrm{~b}$ mostram que os valores vão de 3,4\% em 2009 a $15,6 \%$ em 2019 , valores que mostram um crescimento do número de publicações na temática de mudanças climáticas no país ("climate change" and "Brazil") ao longo do período em estudo (Fig. 7b).

\subsection{Resultados obtidos na base SciELO}

Encontram-se na Fig. 8a os resultados das pesquisas na base SCiELO com os termos "climatology" and "Brazil" e "climatology", observa-se que foram encontradas 37 (trinta e sete) publicações (artigos) no período, com média de 3,4 publicações por ano. São publicações nessa temá-

(a)

\section{Scopus ${ }^{\circledR}$}

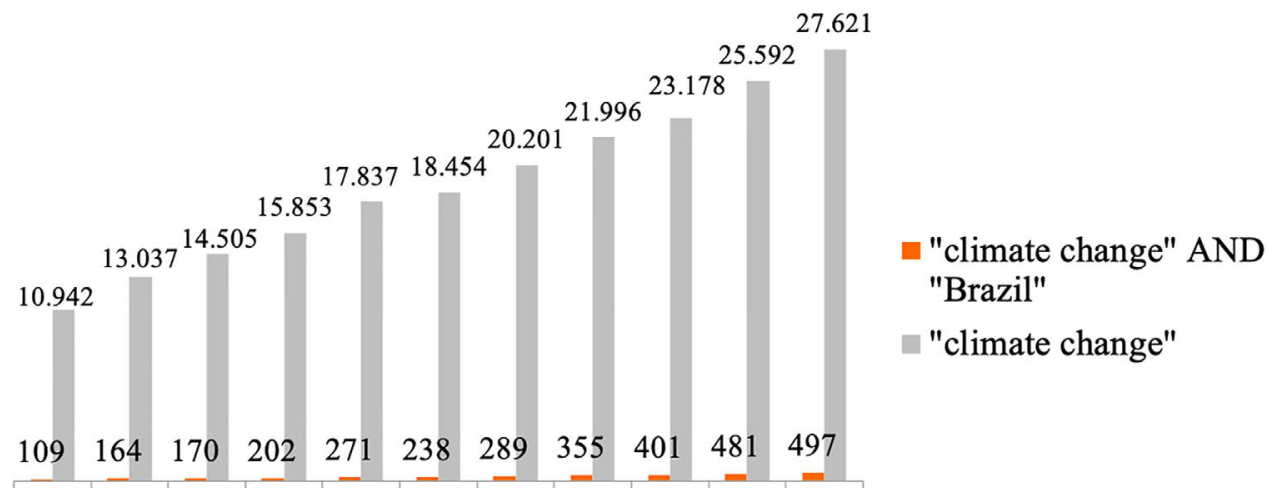

20092010201120122013201420152016201720182019

*Total de publicações "climate change" - 209.216

**Total de publicações "climatology"AND "Brazil" - 3.177

(b)

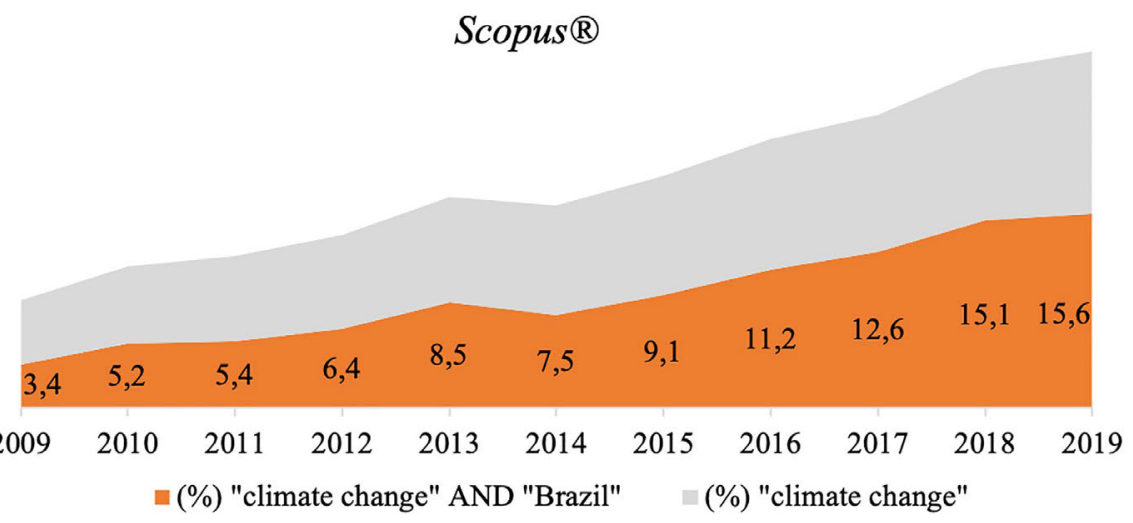

Figura 7 - Resultados obtidos usando-se os termos "climate change" and "Brazil" e "climate change" (2009-2019) - Scopus. Fonte: Portal de Periódicos da Capes (2020).
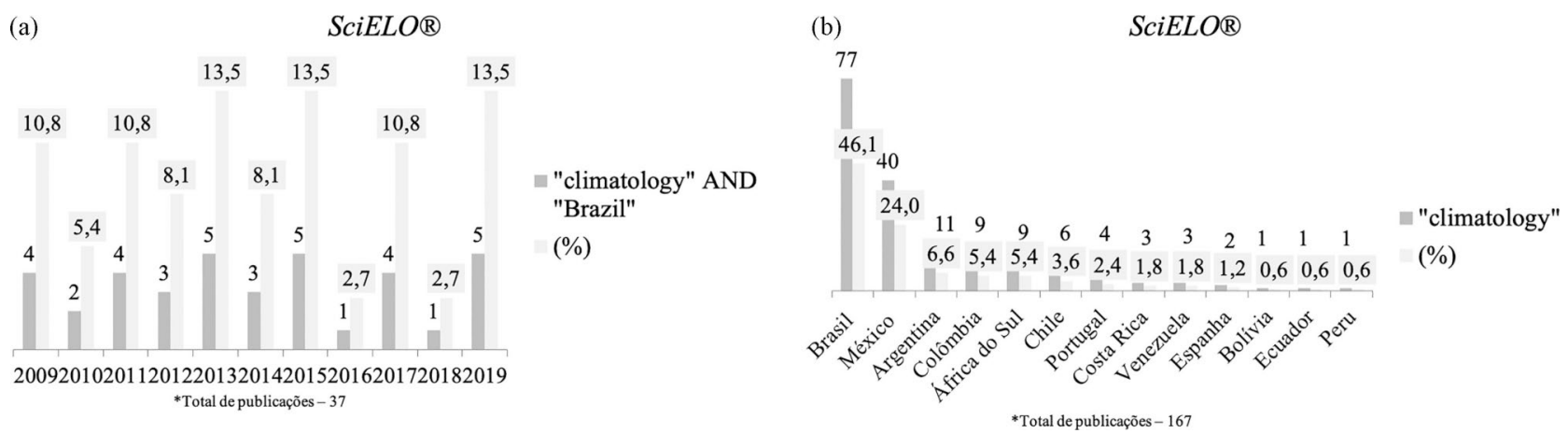

Figura 8 - Resultados obtidos usando-se os termos "climatology" and "Brazil" e "climatology" (2009-2019) - SciELO. Fonte: Portal de Periódicos da Capes (2020). 
tica que estão nas diversas áreas do conhecimento definidas na árvore de especialidades do conhecimento do CNPq: Ciências Exatas e da Terra (14), Ciências Agrárias (13), Ciências Sociais Aplicadas (2), Ciências Biológicas (2), Engenharias (2), Ciências Humanas (2), Multidisciplinar (1), Ciências da Saúde (1).

O Brasil lidera as publicações na temática de climatologia, são encontradas no período em estudo (SciELO) 77 (setenta e sete) publicações brasileiras, correspondente a $46,1 \%$ do total de publicações encontradas, na sequência tem-se as publicações dos mexicanos e argentinos, correspondente a $24 \%$ e $6,6 \%$, respectivamente (Fig. 8b).

Estão listados na Tabela 1 os periódicos da SciELO mais procurados para a publicação dos estudos (artigos) por pesquisadores que se dedicam as questões associadas as temáticas da área de climatologia, destaca-se a Revista Brasileira de Meteorologia (RBMET) com aproximadamente $38 \%$ dos artigos publicados.

Foram encontradas 2.250 (duas mil duzentos e cinquenta) publicações na pesquisa com os termos "climate change". Em geral, tem-se um aumento das publicações durante o período em estudo, variando de 3,3\% em 2009 até $12,6 \%$ em 2019, com destaca-se para o ano de 2016 que apresenta 13,7\% do total de publicações (Fig. 9a).

Tabela 1 - Lista de periódicos que estão publicados os artigos encontrados na pesquisa com os termos "climatology" and "Brazil" na SCiELO.

\begin{tabular}{lc}
\hline Periódico & Artigos \\
\hline Revista Brasileira de Meteorologia (RBMET) & 11 \\
Revista Brasileira de Engenharia Agrícola e Ambiental & 4 \\
Bragantia & 3 \\
Brazilian Journal of Biology & 2 \\
Ambiente Construído & 1 \\
Anais da Academia Brasileira de Ciências & 1 \\
Atmósfera & 1 \\
Brazilian Journal of Oceanography & 1 \\
Ciência e Agrotecnologia & 1 \\
Engenharia Agrícola & 1 \\
Finisterra - Revista Portuguesa de Geografia & 1 \\
Revista de Geografia e Ordenamento do Território & 1 \\
Jornal Vascular Brasileiro & 1 \\
Journal of Aerospace Technology and Management & 1 \\
Mercator (Fortaleza) & 1 \\
Pesquisa Agropecuária Brasileira & 1 \\
Pesquisa Agropecuária Tropical & 1 \\
Revista Ambiente \& Água & 1 \\
Revista Ciência Agronômica & 1 \\
Revista de Gestão Costeira Integrada & 1 \\
Scientia Agricola & 1 \\
\hline Font: Potal Perodicos da Capes (2020).
\end{tabular}

Fonte: Portal de Periódicos da Capes (2020).
As publicações encontradas nesse estudo na temática mudanças climática, em maioria são artigos científicos que apresentam resultados de estudos e pesquisas interdisciplinares que relacionam os possíveis cenários de emissão de gases de efeito estufa, bem como os cenários de variabilidade climática associados ao aumento de temperatura do ar e da ocorrência de eventos extremos que causam chuvas torrenciais em diversas regiões, com questões diversas das áreas de: Ciências Agrárias; Ciências Biológicas; Ciências Sociais Aplicadas; Ciências Humanas; Ciências da Saúde e Engenharias.

Refinando-se a pesquisa para o país com os termos "climate change" and "Brazil" encontram-se 309 (trezentos e nove) publicações (artigos), com $87,4 \%$ dessas publicações em periódicos brasileiros, sendo $4,2 \%$ das publicações na fronteira do conhecimento com a saúde pública, resultados mostrados na Fig. $9 \mathrm{~b}$.

Os resultados apresentados mostram que, assim como observado nas pesquisas realizadas nas outras bases de indexação de periódicos (Science Direct e Scopus), as publicações na temática das mudanças climáticas ("climate change") superam em números as publicações encontradas nas pesquisas com o termo "climatology" ou com os termos "climatology" and "Brazil".

\subsection{Qualificação dos periódicos segundo o Qualis CAPES (2013-2016)}

Os resultados apresentados (Figs. 10a e 10 b) mostram que 7 (sete) dos 11 (onze) periódicos que contém o

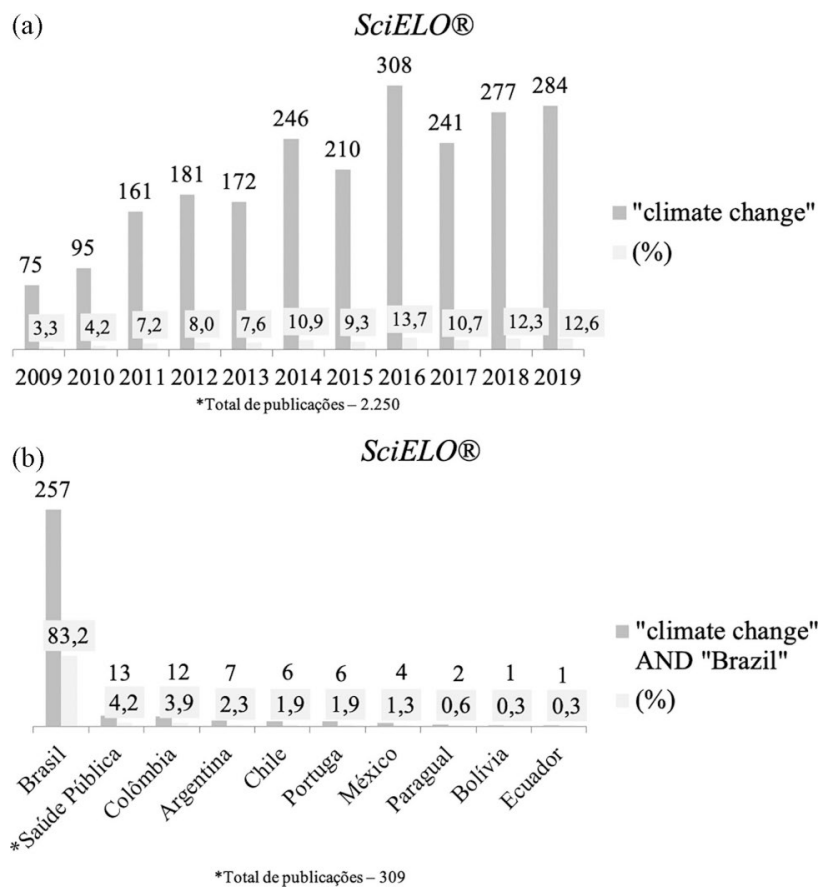

Figura 9 - Resultados obtidos usando-se os termos "climate change" e "climate change" and "Brazil" (2009-2019) - SciELO. Fonte: Portal de Periódicos da Capes (2020). 
(a)
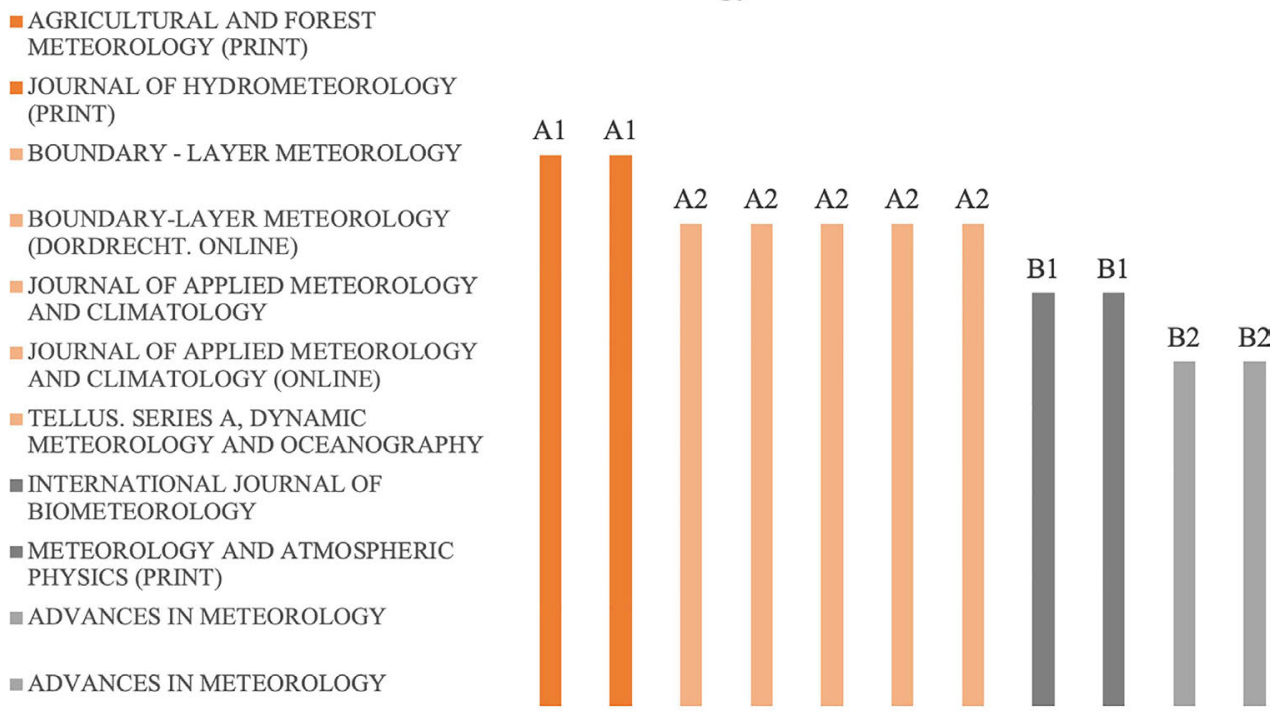

$$
\begin{gathered}
\text { Qualis CAPES (evento } 2013 \text { - 2016) - Geociências } \\
\text { "Climatology" }
\end{gathered}
$$
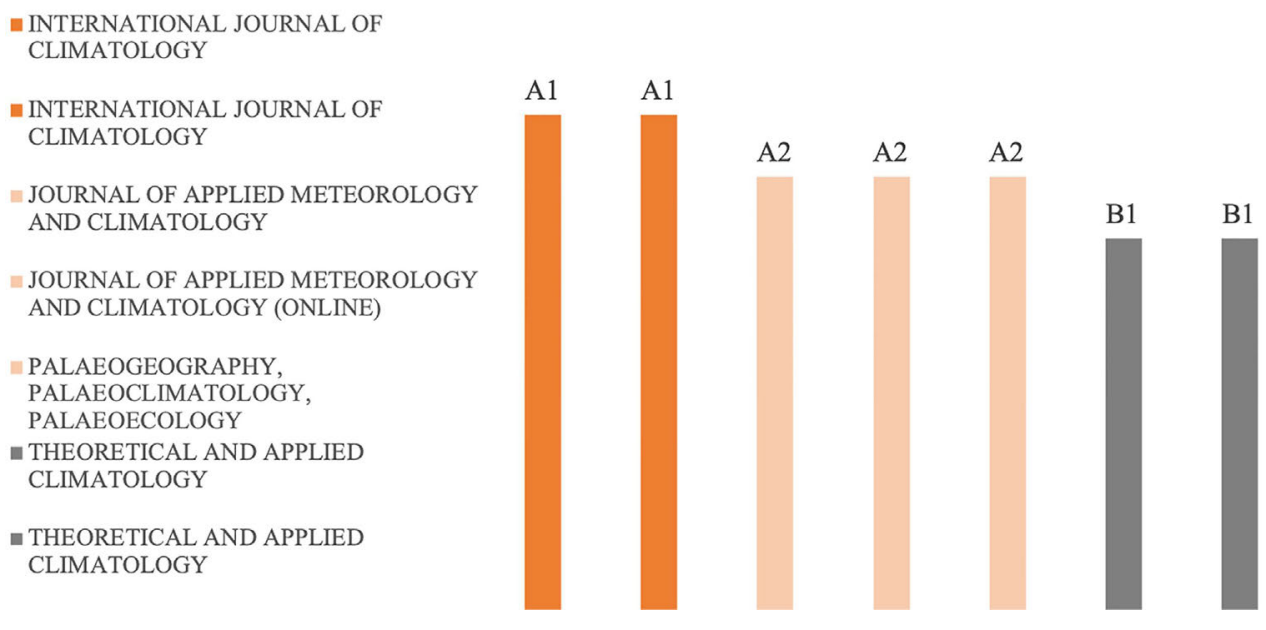

Figura 10 - Classificação dos periódicos indexados ("Meteorology" e "Climatology") em bases internacionais no Qualis CAPES (evento 2013-2016) Área de Geociências. Fonte: Portal de Periódicos da Capes (2020).

termo "Meteorology" em seu título estão classificados no extrato A (A1 e A2) do Qualis CAPES e que 4 (quatro) desses estão no extrato B, classificados como B1 e B2. Em adição, mostram que quando se pesquisa o termo "Climatology" se encontram 7 (sete) periódicos e que 5 (cinco) desses estão no extrato A (A1 e A2), os demais estão classificados no extrato B (B1).

Observa-se que 12 (doze) dos 18 (dezoito) periódicos que contém os termos "Meteorology" e "Climatology", correspondente a $67 \%$ dos periódicos possíveis de publicação dos artigos produzidos na temática em estudo, são qualificados como de excelência para a publicação pelo Qualis CAPES, consequentemente, apresentam maior pontuação para os currículos dos pesquisadores e para a classificação dos programas de pós-graduação que estão aprovados para funcionamento pela CAPES.

Em adição, os resultados mostram (Fig. 11a) que se encontram apenas dois periódicos nacionais quando se pesquisa com os termos em língua portuguesa "Meteorologia" e "Climatologia". A Revista Brasileira de Meteorologia (RBMET) da Sociedade Brasileira de Meteorologia (SBMET), classificada no extrato B (B2) e a Revista Brasileira de Climatologia que tem classificação B5. Destaca-se que a RBMET é indexada na base SciELO e, como mostrado acima, apresenta-se como o principal veículo de divulgação científica quando se pesquisa sobre assunto "Climatologia" nessa base indexadora. 
(a) Qualis CAPES (evento 2013 - 2016) - Geociências

"Meteorologia" - "Climatologia"

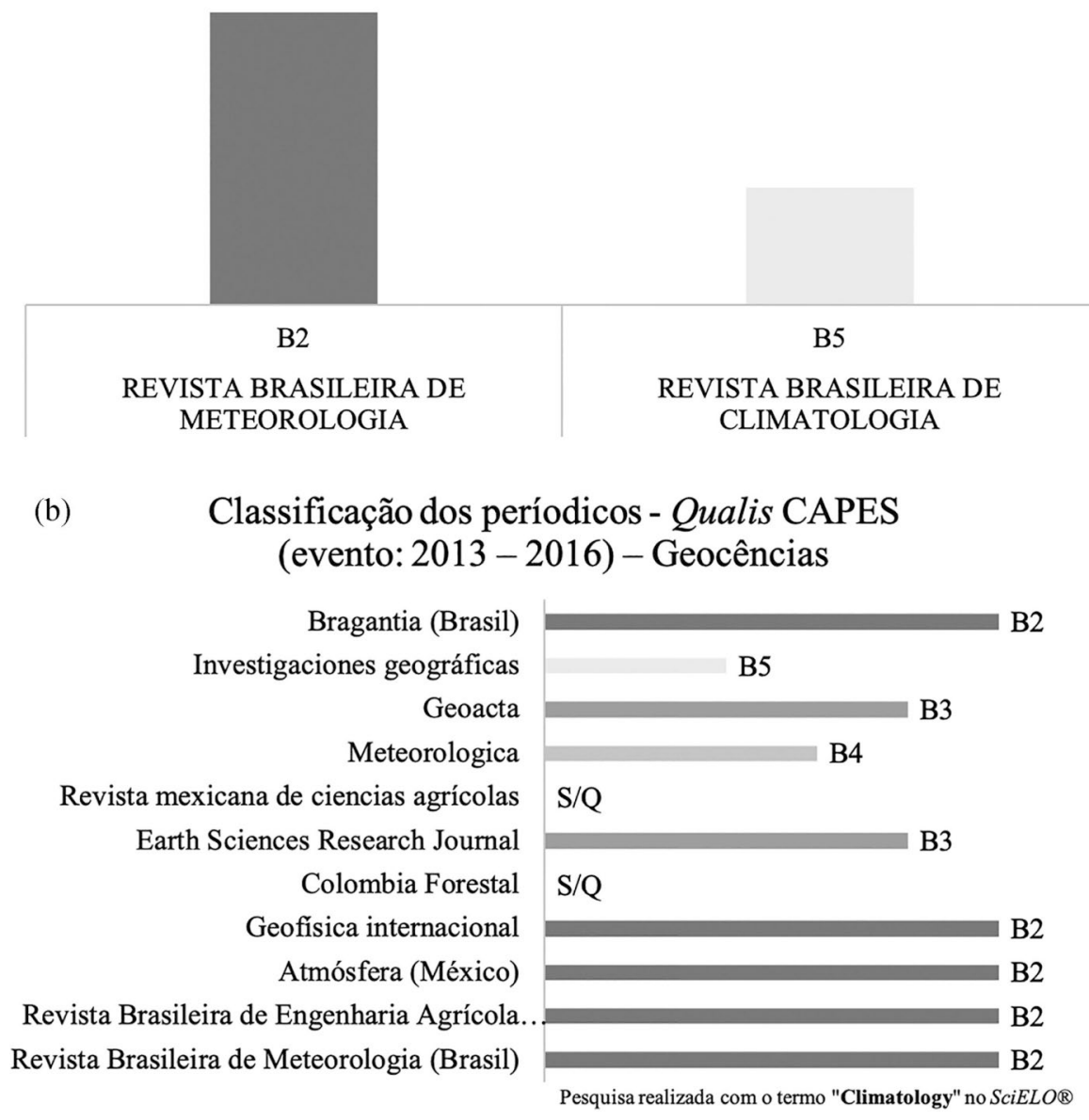

Figura 11 - Classificação dos periódicos indexados ("Meteorologia" e "Climatologia”) em bases internacionais no Qualis CAPES (evento 2013-2016) Área de Geociências. Fonte: Portal de Periódicos da Capes (2020).

Por fim, foi avaliado a classificação no Qualis CAPES (evento 2013-2016) - Área de Geociências - dos principais periódicos que aparecem na pesquisa com o termo "Climatology" and "Brazil" na base SciELO. Os resultados mostram (Fig. 11b) que as revistas indexadas nessa base não são classificadas no extrato de excelência do Qualis CAPES na Área de Geociências, são classificadas no extrato $B$ e algumas sequer aparecem na classificação (S/Q).

\section{Conclusões}

Os resultados das análises das pesquisas realizadas na base indexadora Science Direct levam a concluir que se encontra maior número de publicações na temática de mudanças climáticas do que em climatologia e que se observa um crescimento na quantidade de publicações ao longo do período em estudo (2009 a 2019), destacando-se de 2016 a 2019.
Em adição, conclui-se que as publicações encontradas nessa base indexadora estão distribuídas em três grandes formas, ou modalidades, de publicação. Destacando-se a publicação na forma de artigos de pesquisa (científicos) que corresponde a $92,9 \%$ das publicações encontradas na área de Climatologia e a 94,2\% quando se refere a temática das mudanças climáticas.

As pesquisas realizadas na base indexadora Scopus revelam que os quantitativos de publicações encontrados são maiores que os encontrados nas outras duas bases indexadoras (Science Direct e SciELO), corresponde a $85,6 \%$ do total de publicações encontrado nas três bases indexadoras de periódicos investigadas nesse estudo.

Em semelhança ao encontrado para a base Science, encontra-se um período de destaque (2016 a 2019) que apresenta aumento de publicações na temática em estudo, em particular para a temática de mudanças climáticas.

Período em que se evidencia a eleição pela comunidade científica mundial da relevância dessa temática para 
o diagnóstico e o enfrentamento das consequências dos desastres naturais, em particular nas comunidades que se encontra em situação de vulnerabilidade socioeconômica e que tem-se produzido além dos artigos científicos, de pesquisas e de revisão, que são publicados em revistas de periódicos, são produzidos livros e comunicações técnicas que são apresentadas e publicadas nos anais de eventos científicos (congressos, conferência e outros), nacionais e internacionais, dedicados a essa temática.

A base indexadora SciELO apresenta o menor número de publicações quando comprada com as demais bases usadas nesse estudo, corresponde apenas a aproximadamente $1 \%$ do total de publicações encontrado nas três bases investigadas.

As publicações de artigos em revistas brasileiras dominam as publicações nessa base indexadora, correspondente a $87,4 \%$ das publicações encontradas. Assim como nas outras bases, tem-se que o maior número de publicações encontradas na temática das mudanças climáticas, destacando-se aumento (maiores desvios percentuais da média) das publicações durante o período a partir de 2014 até 2019.

Ressalta-se que as publicações encontradas nessa temática na (SciELO) são artigos científicos que apresentam resultados de estudos e pesquisas interdisciplinares e que trazem reflexões de questões diversas relacionadas com as áreas afins definidas na árvore de especialidades do conhecimento do CNPq: Ciências Agrárias; Ciências Biológicas; Ciências Sociais Aplicadas; Ciências Humanas; Ciências da Saúde e Engenharias.

Em relação a determinação da qualificação, ou seja, da classificação dos periódicos que agregam as publicações da temática em estudo, conclui-se que a maioria (67\%) dos periódicos dedicados as áreas de Meteorologia e Climatologia, que agregam as publicações na temática de mudanças climáticas, encontram-se no extrato de excelência (A) do Qualis CAPES.

Analisando-se na base SciELO especificamente os periódicos que apresentam os maiores quantitativos de publicações na temática do estudo, conclui-se que as revistas indexadas nessa base não são classificadas no extrato de excelência (A) do Qualis CAPES na Área de Geociências. Essas são, em sua totalidade, classificadas no extrato $B$ e algumas sequer aparecem na classificação $(\mathrm{S} /$ Q) desse sistema de classificação de periódicos.

\section{Referências}

CAMPELLO, B.S.; CENDÓN, B.V.; KREMER, J.M. (orgs.) Fontes de Informação Especializada: Características e Utilização. Belo Horizonte: UFMG, 1993.

CAMPELLO, B.S. (orgs.) Fontes de informação para pesquisadores e profissionais. Belo Horizonte: Ed. UFMG, 2000.

DROESCHER, F.D.; SILVA, E.L. Open Access and the use of scientific information. Investigación Bibliotecológica:
Archivonomía, Bibliotecología e Información, v. 29, n. 65, p. 161-194, 2015.

ERDMANN, A.L.; MARZIALE, M.H.P.; PEDREIRA, M.L.G.; LANA, F.C.F.; PAGLIUCA, L.M.F.; PADILHA, M.I.; FERNANDES, J.D. A avaliação de periódicos científicos Qualis e a produção brasileira de artigos da área de enfermagem. Rev Latino-americana de Enfermagem, v. 17, n. 3, p. 403-409, 2009.

FREITAS, J.L.; FÁBIO, S.R.; SANDRA, M. Estudos métricos da informação em periódicos do Portal SciELO: Visibilidade e impacto na Scopus e Web of Science. Palabra Clave, v. 6, n. 2, p. 1-12, 2017.

LANCASTER, F.W. Indexação e Resumos: Teoria e Prática. Brasília: Briquet de Lemos, 2004.

MEADOWS, A.J. A Comunicação Científica. Brasília: Briquet de Lemos, 268 p., 1999.

MUGNAINI, R.; DAMACENO, R.J.P.; DIGIAMPIETRI, L.A.; MENA-CHALCO, J.P. Panorama da produção científica do Brasil além da indexação: Uma análise exploratória da comunicação em periódicos. Transformação, v. 31, n. 1, p. 1-15, 2019.

OLIVER, F.C. Pesquisa e produção bibliográfica em terapia ocupacional: Contribuições ao debate sobre parâmetros de avaliação da produção acadêmica brasileira. Revista de Terapia Ocupacional, v. 19, n. 2, p. 108-120, 2008.

PORTAL DE PERIÓDICOS DA CAPES. Disponível em: www. periodicos.capes.gov.br. Acesso em 14 de abril de 2021.

PUCCINI, L.R.S.; GIFFONI, M.G.P.; SILVA, L.F.; UTAGAWA, C.Y. Comparativo entre as bases de dados PubMed, SciELO e Google Acadêmico com o foco na temática educação médica. Cadernos UniFOA, v. 10, n. 28, p. 75-82, 2015.

RUSSELL, J.M. Scientific communication at the beginning of the twenty-first century. International Social Science Journal, v. 53, n. 168, p. 271-282, 2001.

Sience Direct. Guia de Referência Rápida. Acesso em 14 de abril de 2021. Disponível em: https:/www.periodicos. capes.gov.br/images/documents/ScienceDirect_Guia\% 20de $\% 20$ refer $\%$ C3\%AAncia $\% 20 \mathrm{r} \% \mathrm{C} 3 \%$ A1 pida 28.07.2016 pdf.pdf

Scopus. Guia de Referência Rápida. Acesso em 14 de abril de 2021. Disponível em: https://www.periodicos.capes.gov.br/ images/documents/Scopus_Guia $\% 20 \mathrm{de} \% 20$ refer $\% \mathrm{C} 3 \%$ AAncia\%20r\%C3\%A1pida_10.08.2016.pdf.

Scopus. Guia de Referência Rápida. Acesso em 14 de abril de 2021. Disponível em: https://www.periodicos.capes.gov.br/ images/documents/Scopus_Guia $\% 20 \mathrm{de} \% 20$ refer $\% \mathrm{C} 3 \%$ AAncia\%20r\%C3\%A1pida_10.08.2016.pdf.

ZIMAN, J. Conhecimento Público. São Paulo: Editora da Universidade de São Paulo, 1979.

\section{Endereços de Internet}

Portal de Periódicos da Capes: www.periodicos.capes.gov.br. SciELO: https://www.scielo.br/.

Plataforma Sucupira: https://sucupira.capes.gov.br/sucupira/.

License information: This is an open-access article distributed under the terms of the Creative Commons Attribution License (type CC-BY), which permits unrestricted use, distribution and reproduction in any medium, provided the original article is properly cited. 